\title{
Hematological Characteristics of COVID-19 Patients Admitted to Fayoum University Hospital
}

\author{
Ahmed A. Gomaa ${ }^{1}$, Essam A. Hassan ${ }^{1}$, Fatma A. Mohammed ${ }^{1}$, Eman E. \\ Mahmoud $^{2}$, and Sara M. Abd Elsatar ${ }^{1}$. \\ 1 Tropical Medicine Department, Faculty of Medicine, Fayoum University \\ ${ }^{2}$ Clinical and chemical pathology Department, Faculty of Medicine, Fayoum \\ University
}

Corresponding author: Ahmed A. Gomaa

E-mail address: Afify1968@yahoo.com

Tel: 01006158187

Fax:

\begin{abstract}
On March $11^{\text {st }}, 2020$, the WHO declared the outbreak of SARS-CoV-2 a global pandemic, reporting community-scale transmissions occurring in every continent outside Antarctica. Infected patients with COVID-19 exhibit a wide spectrum of symptoms and severity; ranging from totally asymptomatic carriers to severe symptoms such as ARDS, septic shock with MOF. ARDS is the most critical clinical manifestation of COVID-19, induced mainly through immune response (i.e., cytokine storm). ACE2 is the main receptor for SARS-COV2, definitively play an important role in the pathogenesis and severity of covid-19 by its wide distribution throughout the body organs and its role in modulating the immune system. Aim of the work was to collect and analyze the hematological
\end{abstract}

characteristics of patients with COVID-19 admitted at FUH. Type of the study: Retrospective cohort study. We performed a retrospective study of the hematological characteristics in confirmed cases with COVID-19 by RT-PCR hospitalized at FUH between April and July 2020. Baseline demographic data of the studied patients. The main gender was male with $69.3 \%$ percentage with mean age $37.4 \pm 15.6$ (between $4^{\text {th }}$ and $6^{\text {th }}$ decade), about $61.4 \%$ of them came from rural areas. Our results revealed that, the predictor importance of CRP; it is directly related to the patient's age, positively affects the duration of hospital stay, fever and to some extent on the $\mathrm{O}_{2}$ saturation /hypoxia. Based on the current study results, we can conclude that: CRP, has perfect accuracy in predicting cases with positive RTPCR for COVID-19 and their severity. 
KEY WORDS: CRP, COVID-19, INTRODUCTION

Since December 2019, several Wuhan city hospitals (Hubei Province, China) have detected unidentified pneumonia, when it was recognized as a novel coronavirus (2019-nCoV). The virus soon spread to other countries and regions within two months [1]. The outbreak has already spread to 220 countries worldwide, infecting more than 173 million individuals and triggering more than 3.7 million fatalities [2]. Egypt announced the detection of the first COVID19 case on the $14^{\text {th }}$ of February 2020 'CO' stands for corona, 'VI' for virus 'D' for disease and '19' for 2019 [2].

Coronavirus is a wide family of viruses that cause diseases ranging from mild colds to more severe diseases, such as Middle East Respiratory Syndrome (MERS) and severe acute respiratory syndrome (SARS). The SARS-COV2 is a modern strain, which has not been previously recognized in humans. On $11^{\text {th }}$ February 2020, the International Committee for Virus Taxonomy released a statement confirming the formal name for the novel virus: "severe acute respiratory syndrome coronavirus 2" (SARS-CoV-2) [3]. prediction

The diagnosis relies on estimating the likelihood of contact with an infected patient, the clinical signs and symptoms, and detecting the viral ribonucleic acid (RNA) by polymerase chain reaction (PCR) in respiratory samples [1].

The full clinical presentation of COVID-19 is still not understood, but among the most common presentations are cough, fever, dyspnea, anosmia and pneumonia. To date, no specific drug has been reported that specifically acts against COVID-19. So, early identification of severity of COVID-19 is very important to enable intervention for the reduction of mortality risk and to predict prognosis [4].

The inflammatory
cytokine storm has been recognized as the primary cause of death in patients with COVID19 [5], which is defined by the excessive and uncontrolled release of pro-inflammatory cytokines, as has been reported in other infections caused by pathogenic coronaviruses [6]. For instance, inflammatory cytokines released by macrophages (interleukin 6 (IL6 ), interleukin10 (IL-10) and tumor necrosis factor $\alpha$ (TNF- $\alpha)$ ) increase in patients with severe 
COVID-19 disease, resulting in damage to the lungs and other organs [5]. Consequently, measurements of plasma inflammatory markers such as Ferritin, Lactate dehydrogenase (LDH) and C-reactive protein (CRP), may predict the disease's seriousness [7]. The aim of the work CRP, predicting cases with positive RT-PCR for COVID-19 admitted at the Fayoum University Hospital (FUH).

\section{PATIENTS AND METHODS}

Type of the study: Retrospective cohort study.

\section{Patients:}

We performed a retrospective study of the hematological characteristics in confirmed cases with COVID-19 by RT-PCR hospitalized at FUH from April to July 2020.

Methods and Data gathering:

All included patients were subjected to the following (as

\section{Results}

This study was started by recruiting 140 post COVID-19 patients; recruitment was done from the internal word and intensive care unit (ICU), Faculty of Medicine, Fayoum University and enrolled between March recorded in Fayoum University hospital electronic files):

Full medical history.

Laboratory investigations including:

CRP.

\section{Statistical analysis:}

All statistical calculations were done using Microsoft Excel version 16 and SPSS (statistic package for the social science version 26.00) statistical program at $0.05,0.01$, and 0.001 levels of probability. Categorical variables were presented as numbers (\%). The Kolmogorov-Smirnov test evaluated the normal distribution of continuous variables. Normally or non-normally distributed continuous parameters were described as mean (standard deviation) or median (interquartile range, IQR). The Kruskal wills through Duncan multiple-range test were shown using Median (IQR). Simple linear regression.

2020 and August 2020 during 1st COVID-19 wave.

Baseline demographic data of the studied patients was shown in table (1); the main gender was male with $69.3 \%$ percentage with mean age $37.4 \pm 15.6$ years (between $4^{\text {th }}$ and $6^{\text {th }}$ decade) and about $61.4 \%$ of them came from rural areas. 
Table 1: Baseline demographic data of the studied cohort:

\begin{tabular}{|c|c|}
\hline Variable & Mean \pm (SD) or number (\%) \\
\hline Age (years) & $37.41( \pm$ SD 15.62) \\
\hline Sex (males) & $97(69.3 \%)$ \\
\hline $\begin{array}{c}\text { Residence (rural } \\
\text { area) }\end{array}$ & $86(61.4 \%)$ \\
\hline
\end{tabular}

\section{Laboratory predictors of the} CRP in mild to moderate cases:

Regarding laboratory predictors of the clinical course of the disease, we used Analysis of variance (ANOVA) as an important method in exploratory and confirmatory data analysis. In addition to ANOVA, we also used a linear model which fits with coefficient estimates and standard errors.

ANOVA is an organization of additive data decomposition. Its sums of squares indicate the variance of each component of the decomposition (or, equivalently, each set of terms of a linear model). In addition to comparisons of mean squares, along with F-tests [or F-like tests allow testing of a nested sequence of models].
We will now discuss the predictive value of different variables ie, CRP in relation to other demographic and clinicolaboratory

constants.

Demographic constants: age, sex, and residence. Clinical constants include medical history: (DM, HTN, cardiac, renal, chest, psychiatry, duration of hospital stay, clinical outcome, ICU admission, presenting symptoms (cough, anosmia, diarrhea, hypoxia, vomiting, dyspnea, fever, and sore throat), complications (sepsis, bacterial chest infection). Laboratory constants (ALT, AST, LDH, urea, creatinine, Hb, WBCs, PLT, PNL, Lymphocytes count \& \%, PT, INR, D-dimer and ferritin) and finally treatment options i.e., zinc and IV steroid intake. 


\section{Predictive value of CRP:}

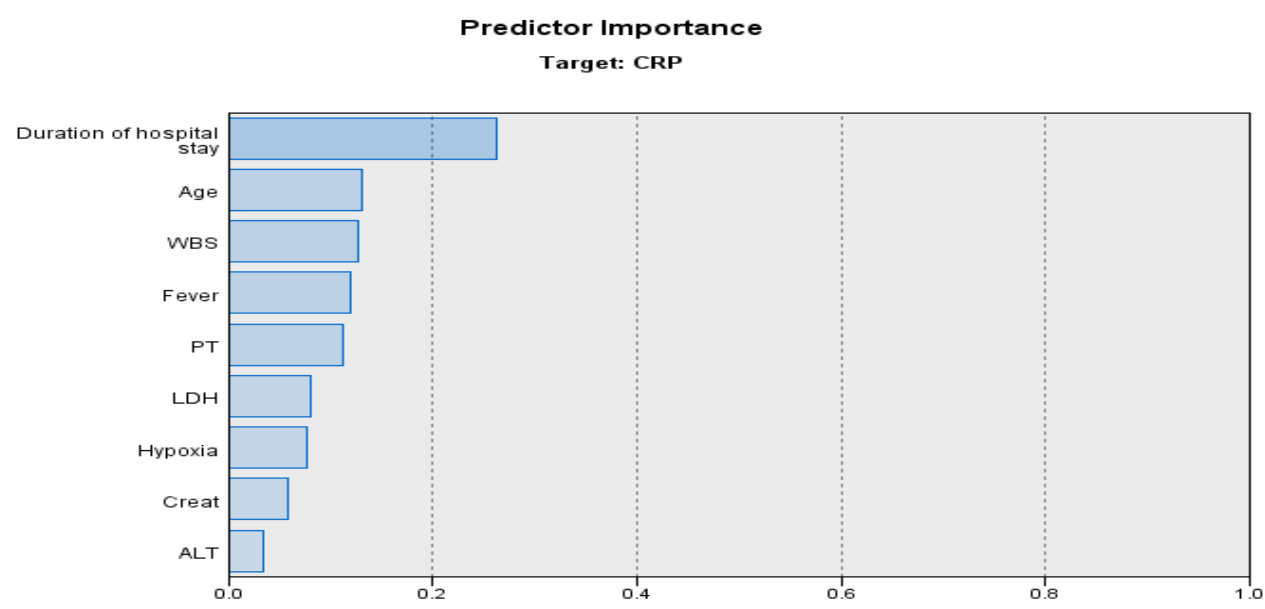

Least Important $\quad$ Most Important

Figure (1): showing the predictor importance of CRP.

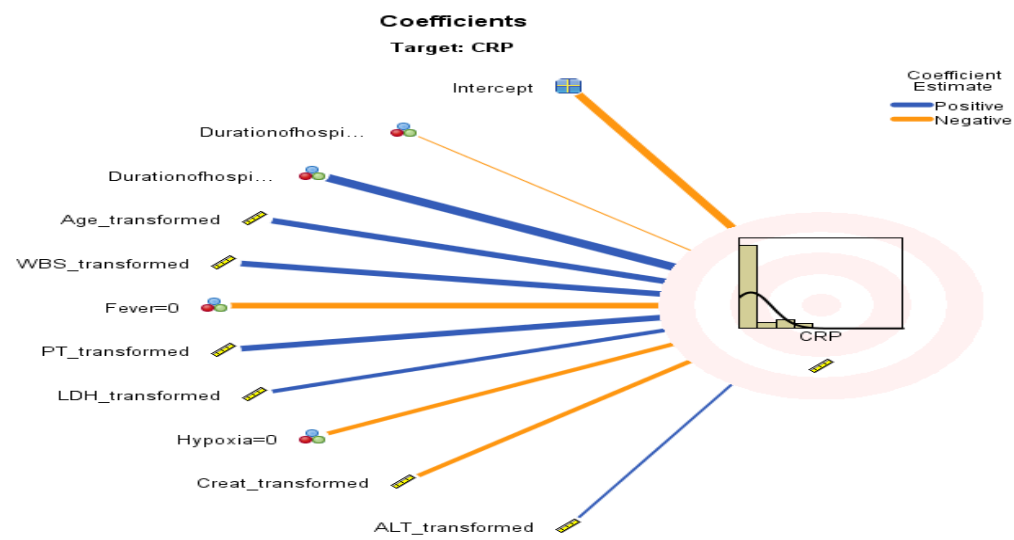

Figure (2): showing the coefficients of CRP.

Figures 1 and 2 show the predictor importance of CRP; it is directly related to the patient's age, positively affects the duration of hospital stay, fever and to some extent on the $\mathrm{O}_{2}$ saturation /hypoxia. A main effect of measuring CRP was found, $\mathrm{F}=5.53, \mathrm{p}<0.001$ as shown in tables $2 \& 3$.

Table 2 Simple linear regression model of CRP as a variable: 


\begin{tabular}{|c|c|c|c|c|}
\hline Model & R & R Square & $\begin{array}{c}\text { Adjusted R } \\
\text { Square }\end{array}$ & $\begin{array}{c}\text { Std. Error } \\
\text { of the } \\
\text { Estimate }\end{array}$ \\
\hline & 0. & 0.70 & 0.574 & 16.975 \\
& 83 & 0 & & 90 \\
& 7 & & & \\
\hline
\end{tabular}

Table 3: ANOVA model of CRP as a variable:

\begin{tabular}{|l|c|c|c|c|c|}
\hline Model & $\begin{array}{l}\text { Sum of } \\
\text { square } \\
\text { s }\end{array}$ & $\begin{array}{c}\text { d } \\
\mathbf{f}\end{array}$ & $\begin{array}{c}\text { Mean } \\
\text { squar } \\
\text { e }\end{array}$ & F & $\begin{array}{c}\text { Signific } \\
\text { ance }\end{array}$ \\
\hline Regress & 65329. & 4 & 1593. & 5. & 0.000 \\
ion & 452 & 1 & 401 & 52 & \\
\hline Residua & 27953. & 9 & 288.1 & 9 & \\
I & 568 & 7 & 81 & & \\
\hline Total & 93283. & 1 & & & \\
& 021 & 3 & 8 & &
\end{tabular}

$F$ value $(F)$, degrees of freedom (df) (numerator, denominator; in parentheses separated by a comma next to $F$ ) and significance level ( $p$ ).

\section{DISCUSSION}

The present study was conducted to study the hematological characteristics of COVID-19 patients and to detect predictors affecting the outcome in patients with the mild-moderate clinical picture.
When reviewing the demographic data of the current study population, there was a male predominance; males were $69.3 \%$ and the mean age was 37.41 years for the whole study group. This match with the known epidemiological features of COVID-19 patients, one of 
them was done by Ahmad, et al. [8] who noticed that the median age of their study cohort was 34 years and $80.9 \%$ were males. It was also similar to those of previous research in China, in which the proportion of infected females was lower than that of infected men [9].

The predominance of male gender in COVID-19 patients could be explained by many theories, such as hormoneregulated expression of genes encoding for SARS-CoV2 entry receptors ACE2 and TMPRSS2 as well as sex hormone-driven innate and adaptive immune responses and immune-aging. Also, the gender-specific lifestyle, health behavior, psychological stress and socioeconomic conditions may have their impact on COVID-19 [10].

Most of the patients came from rural areas $(61.4 \%)$ and this could be explained by many factors as the overcrowding of rural areas, lack of social distancing and precautionary measures which are significant risk factors for rapid transmission of SARS-CoV-2 infection, which was supported by Guo, et al. [11] who found that several rural communities had high incidence rate of covid-19 which was related to the presence of a COVID-19-positive individual at highly attended funerals. They also found that in countries such as India, 25\% of COVID-19related deaths have occurred in rural and suburban districts.

Regarding laboratory predictors of COVID-19, we found that CRP, LDH, ferritin, D-dimer and lymphocytes percentages were the most significant ones.

CRP is an acute-phase inflammatory protein, a highly conserved plasma protein that was initially discovered in 1930 by Tillet and Francis while investigating the sera of patients suffering from the acute stage of Pneumococcus infection and was named for its reaction with the capsular (C)-polysaccharide of Pneumococcus [12]. It is synthesized primarily in liver hepatocytes but also by smooth muscle cells, macrophages, endothelial cells, lymphocytes, and adipocytes.

As an acute-phase protein, the plasma concentration of CRP deviates by at least $25 \%$ during inflammatory disorders [13].

The highest concentrations of CRP are found in serum, with some bacterial infections increasing levels up to 1,000-fold [14]. 
Regarding CRP, in our study it had significantly risen in most patients. Retrospective singlecenter research in Wuhan, China, has emphasized the use of CRP in COVID-19 patients. The majority of patients in the severe group have considerably higher values $(57.9 \mathrm{mg} / \mathrm{L})$ [6]. A second retrospective cohort research found that individuals with CRP levels more than $41.8 \mathrm{mg} / \mathrm{L}$ were more likely to proceed to severe COVID-19 disease [15]. CRP levels seemed to be a robust indication of the presence and severity of COVID-19 infection in both studies which support our results.

\section{CONCLUSION}

CRP, has perfect accuracy in predicting cases with positive RTPCR for COVID-19 and their severity.

\section{REFERENCES}

1. Singhal, T. "A review of coronavirus disease-2019 (covid-19)." Indian J Pediatr 87 (2020): 281-86. 10.1007/s12098-02003263-6.

https://www.ncbi.nlm.nih.gov/pubmed/32 $\underline{166607 .}$.

2. WHO. "Draft landscape of covid-19 candidate vaccines.

https://www.who.int/publications/m/item/d raft-landscape-of-covid-19-candidatevaccines.Date. (2021):

3. Lescure, F., L. Bouadma, D. Nguyen, M. Parisey, P. Wicky, S. Behillil, A. Gaymard, M. Bouscambert-Duchamp, F. Donati and Q. Le Hingrat. "Clinical and virological data of the first cases of covid19 in europe: A case series." The Lancet Infectious Diseases 20 (2020): 697-706.

4. Xu, Z., L. Shi, Y. Wang, J. Zhang, L. Huang, C. Zhang, S. Liu, P. Zhao, H. Liu, L. Zhu, et al. "Pathological findings of covid-19 associated with acute respiratory distress syndrome." The Lancet Respiratory Medicine 8 (2020): 420-22. 10.1016/s2213-2600(20)30076-x.

5. Chen, G., D. Wu, W. Guo, Y. Cao, D. Huang, H. Wang, T. Wang, X. Zhang, H. Chen, $\mathrm{H}$. $\mathrm{Yu}$, et al. "Clinical and immunological features of severe and moderate coronavirus disease 2019." $J$ Clin Invest 130 (2020): 2620-29. 10.1172/JCI137244.

https://www.ncbi.nlm.nih.gov/pubmed/32 $\underline{217835}$.

6. Qin, C., L. Zhou, Z. Hu, S. Zhang, S. Yang, Y. Tao, C. Xie, K. Ma, K. Shang, W. Wang, et al. "Dysregulation of immune response in patients with coronavirus 2019 (covid-19) in wuhan, china." Clin Infect Dis 71 (2020): 762-68. 10.1093/cid/ciaa248.

https://www.ncbi.nlm.nih.gov/pubmed/32 161940.

7. Zhao, J., Y. Yang, H. Huang, D. Li, D. Gu, X. Lu, Z. Zhang, L. Liu, T. Liu, Y. Liu, et al. "Relationship between the abo blood group and the covid-19 susceptibility." Clin Infect Dis (2020): 10.1093/cid/ciaa1150.

https://www.ncbi.nlm.nih.gov/pubmed/32 750119.

8. Ahmad, M., B. M. Beg, A. Majeed, S. Areej, S. Riffat, M. A. Rasheed, S. Mahmood, R. M. Z. Mushtaq and M. A. Hafeez. "Epidemiological and clinical characteristics of covid-19: A retrospective multi-center study in pakistan." Front Public Health 9 (2021): 644199. 10.3389/fpubh.2021.644199. https://www.ncbi.nlm.nih.gov/pubmed/33 937174.

9. Al-Qahtani, M., S. AlAli, A. AbdulRahman, A. Salman Alsayyad, S. Otoom and S. L. Atkin. "The prevalence of asymptomatic and symptomatic covid19 in a cohort of quarantined subjects." Int $J$ Infect Dis 102 (2021): 285-88. 
10.1016/j.ijid.2020.10.091.

https://www.ncbi.nlm.nih.gov/pubmed/33

157290.

10. Gebhard, C., V. Regitz-Zagrosek, H. K. Neuhauser, R. Morgan and S. L. Klein. "Impact of sex and gender on covid-19 outcomes in europe." Biol Sex Differ 11 (2020): 29. 10.1186/s13293-020-00304-9. https://www.ncbi.nlm.nih.gov/pubmed/32 450906.

11. Guo, Y., W. Qin, Z. Wang and F. Yang. "Factors influencing social distancing to prevent the community spread of covid-19 among chinese adults." Prev Med 143 (2021): 106385 .

10.1016/j.ypmed.2020.106385.

https://www.ncbi.nlm.nih.gov/pubmed/33 359017.

12. Tillett, W. S. and T. Francis. "Serological reactions in pneumonia with a non-protein somatic fraction of pneumococcus." $J$ Exp Med $52 \quad$ (1930): $561-71$. 10.1084/jem.52.4.561.

https://www.ncbi.nlm.nih.gov/pubmed/19 $\underline{869788 .}$.

13. Gabay, C. and I. Kushner. "Acute-phase proteins and other systemic responses to inflammation." N Engl J Med 340 (1999): 448-54. 10.1056/NEJM199902113400607. https://www.ncbi.nlm.nih.gov/pubmed/99 71870.

14. Sproston, N. R. and J. J. Ashworth. "Role of c-reactive protein at sites of inflammation and infection." Front Immunol 9 (2018): 754. 10.3389/fimmu.2018.00754.

https://www.ncbi.nlm.nih.gov/pubmed/29 706967.

15. Liu, F., L. Li, M. Xu, J. Wu, D. Luo, Y. Zhu, B. Li, X. Song and X. Zhou. "Prognostic value of interleukin-6, creactive protein, and procalcitonin in patients with covid-19." J Clin Virol 127 (2020): 104370 .

10.1016/j.jcv.2020.104370. https://www.ncbi.nlm.nih.gov/pubmed/32 $\underline{344321 .}$. 